\title{
A Solution for the Dropout Problem in Adaptive Cruise Control Range Sensors
}

\author{
Bongsoo Son ${ }^{1}$, Taehyung $\mathrm{Kim}^{2}$, and YongEun $\mathrm{Shin}^{3}$ \\ ${ }^{1}$ Dept. of Urban Plan. and Eng., Yonsei Universiy, Seoul, Korea \\ sbs ayonsei.ac.kr \\ ${ }^{2}$ Adavnced Trans. Tech. Research Center, The Korea Trans. Institute, Korea \\ thkimakoti.re.kr \\ ${ }^{3}$ Dept. of Urban Eng., Dong-Eui Univ., Busan, Korea \\ yshin@deu.ac.kr
}

\begin{abstract}
At the transition from a tangent section to a curved section, it is possible for a following vehicle with adaptive cruise control in car-following mode to lose track of the lead vehicle. This occurs because the lead vehicle enters the curve and its path diverges from the axis of the following vehicle, yet the following vehicle does not yet have lateral acceleration information that would induce its range sensor to bend according to the curvature of its own path. This is a temporary situation, but one that could have an impact on cruise control safety and appropriate algorithms. In this paper, the conditions of time and distance that produce this circumstance are derived. Examples are given using typical values of roadway and vehicle parameters. Finally, some conclusions regarding possible solutions are offered.
\end{abstract}

\section{Introduction}

Correct functioning of an adaptive cruise control system is predicated on the assumption that the following vehicle can continually acquire range information from the vehicle in front of it. This is important both when the subject vehicle is traveling at its desired speed and is alert to potential conflicts in front of it, and while it is in a "carfollowing" mode because a leading vehicle is obstructing its ability to travel at its own desired speed. The acquisition of range information (and any of its derivatives, including relative velocity and relative acceleration) is accomplished via electromagnetic sensor, most often employing infrared radar or laser technology. A variety of sensors are available, and testing regimes have been established to ensure that they perform adequately [1]. For our purposes, the sensor can be visualized in plan view as a beam with a given angular range $\theta$.

Several rangefinder sensors, such as the Omron units employed in some Nissan ACC systems, employ multiple beams. Primarily, this capability is used to support sensing of lane-changing and other potential conflicts outside the lane of the subject vehicle. Each beam has an aiming angle and included angle similar to $\theta$, and each could be analyzed individually using the methods in this paper. 
ACC is becoming a more popular accessory in cars, following the traditional trajectory of new technology, which is to filter its way down from the most expensive models to the lesser so. It is currently marketed as a convenience system rather than a safety aid [2]. This is due in part to the fact that its operation is not yet robust. This paper is concerned with a specific instance where the intended functionality of the ACC range sensor can be hampered, due to a combination of vehicle locations, speeds, and roadway geometry. The next section describes, qualitatively, a number of known issues from the ACC literature. Following that, we present the quantitative methodology for determining the specific circumstances under which rangefinder dropouts can occur at curve transitions, and some examples with specific parameter values. This is followed by a discussion of how this and other sensor issues relate to the design of ACC strategies and algorithms. Finally, some conclusions and suggestions for future research are offered.

\section{Target Determination in Circular Curve}

One of the interesting challenges of ACC control lies in the fact that when both the subject vehicle and the target vehicle are on a curve, the target vehicle is not directly in front of the subject vehicle, as measured along its own axis. To counter this, most ACC sensors have the ability to deflect their beams at appropriate angles. The determination of the proper angle requires an estimate of the curvature of the road being traversed, which is accomplished via a combination of an on-board yaw rate sensor and feedback from the vehicle's speed sensor.

What is assumed in this calculation, however, is that the subject vehicle is on the curve, allowing it to measure its own lateral acceleration. During the transition from a tangent section to a curved section, however, the lead vehicle enters the curve first, and it might be possible for it to turn out of the path of the sensor beam before the following vehicle is even aware that a curve is coming [1]. In their report [3] on a major demonstration project in the U.S., General Motors acknowledges that curve entry-exit transitions present a challenge to target acquisition and tracking in an ACC system. The issue is more complicated than simply losing track of the vehicle - from the perspective of the rangefinder in the following vehicle (if it is of the multi-beam variety equipped to take such measurements), this maneuver might just as easily resemble a lane change. As a result, if the following vehicle were traveling below its desired speed, it might interpret this as a circumstance where it would be safe to accelerate back to its desired speed. In fact, however, it would soon discover, once it was on the curve, that the lead vehicle did not change lanes, and therefore because of its own acceleration, the safe following distance had been compromised. Other issues related to losing track of an appropriate target, or acquiring a stationary target incorrectly, have been documented in [1, 4] and elsewhere. The methodological portion of this paper is concerned only with the issue of temporary losing track of a lead vehicle at a curve transition, although the geometric procedure can be adapted to other problems.

It should be noted that a similar problem might arise with vertical curves. In this case, the "visibility" of the lead vehicle might be hampered. In general, particularly on high-speed facilities where ACC systems are currently most useful, vertical 
curvature tends to have less of an egregious effect than horizontal. Nevertheless, this is probably an important issue, although it is not treated in the scope of this paper.

We present the geometry of this situation in a way that enables to determine, as a function of the speed of the vehicles (and therefore the safe following distance), the distance and time that the following vehicle is on the tangent, after losing track of the target vehicle, but before entering the curve where it might re-acquire. It is this interval that is most dangerous, and it might be wise to not attempt to accelerate during this time.

\section{Geometric Derivation}

It is assumed that the horizontal alignment consists only of a smooth series of connected tangent sections and circular curves. While clothoid spirals are also used frequently for curve transitions, they do not lend themselves to closed-form geometric calculations [5]. Furthermore, their effect is to reduce the rate of change of curvature and spread it over a longer distance, giving a following vehicle more time to enter the curve and become aware of the new circumstances. Thus, the worst case-scenario is a direct tangent-to-circular arc transition, so spirals will not be considered in this paper. Figure 1 shows the geometry of tangent and circular curve sections. In the derivation that follows, coordinates are shown in vector representation for conciseness.

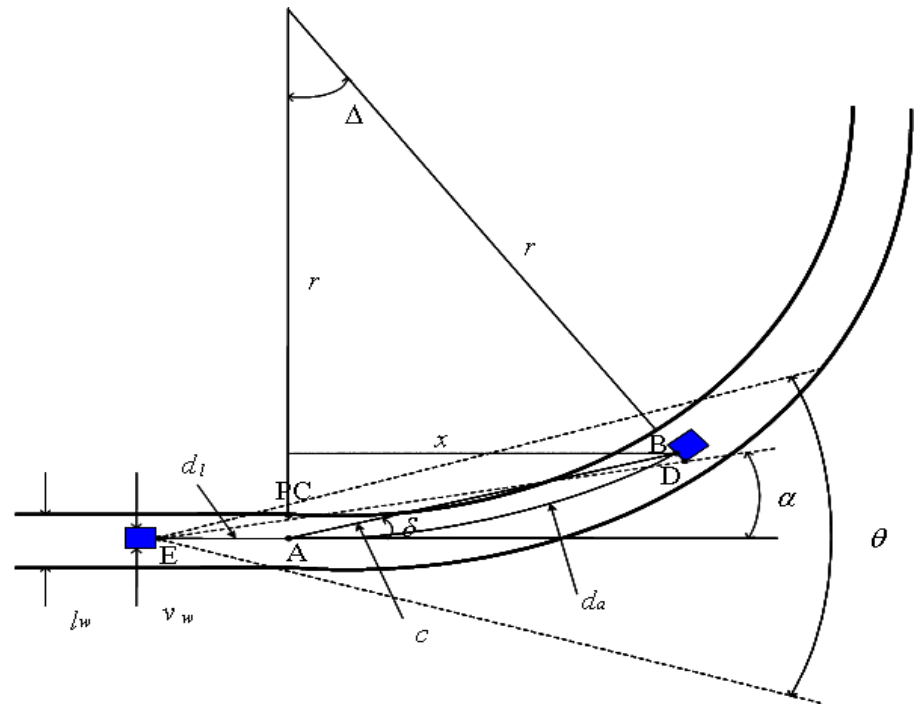

Fig. 1. Geometry of tangent and circular curve sections

The origin of the coordinate system in the figure is at the point $\mathrm{PC}$, which is the point of curvature, or transition from the tangent to the circular curve. The circular arc has radius $r$. The safe following distance between the two vehicles is given by $d$, which is then partitioned into $d=d_{a}+d_{l}$, where $d_{a}$ is the distance along the arc of the 
center of the vehicle travel lane, and $d_{l}$ is the remaining distance, which is apportioned to the tangent. While $d$ might be given exogenously, we will also show an example where it is a commonly used function of the vehicle speed $v$. The included angle $\Delta$, on the circular arc, between its start and the location of the lead vehicle, is given by

$$
\Delta=\frac{2 d_{a}}{2 r+l_{w}}
$$

where $l_{w}$ is the lane width. Throughout this paper, all angles are given in radians.

The point $\mathbf{A}$ is the middle of the driving lane at the curve transition; it is given by

$$
\mathbf{A}=\left[\begin{array}{ll}
\frac{l_{w}}{2} & 0
\end{array}\right]^{\mathrm{T}}
$$

The point $\mathbf{B}$ is the middle of the driving lane at the rear of the lead vehicle. To find this point, first we find the length $C$, of the chord $\mathrm{AB}$ :

$$
c=2\left(r+\frac{l_{w}}{2}\right) \sin \frac{\Delta}{2}=\left(2 r+l_{w}\right) \sin \left(\frac{d_{a}}{2 r+l_{w}}\right)
$$

The point $\mathrm{B}$ is then determined by

$$
\mathbf{B}=\mathbf{A}+c R(\delta)\left[\begin{array}{ll}
0 & 1
\end{array}\right]^{\mathrm{T}}
$$

where $R(\delta)$ is the transformation matrix that effects a counter-clockwise rotation about the origin through an angle of $\delta$ radians (for examples of this form of vector algebra for highway design purposes, see [5,6]), and is given by

$$
R(\delta)=\left[\begin{array}{cc}
\cos \delta & -\sin \delta \\
\sin \delta & \cos \delta
\end{array}\right]
$$

and $\delta$ is the deflection angle (measured in radians, counter-clockwise from the positive abscissa) of the line that is tangent to the circular curve. This can also be written

$$
\delta=\cos ^{-1}\left(\frac{x}{c}\right)
$$

where

$$
x=\left(r+\frac{l_{w}}{2}\right) \sin \Delta
$$

Hence $R(\delta)$ is given by

$$
R(\delta)=\left[\begin{array}{cc}
\cos \left(\cos ^{-1}\left(\frac{x}{c}\right)\right) & -\sin \left(\cos ^{-1}\left(\frac{x}{c}\right)\right) \\
\sin \left(\cos ^{-1}\left(\frac{x}{c}\right)\right) & \cos \left(\cos ^{-1}\left(\frac{x}{c}\right)\right)
\end{array}\right]=\left[\begin{array}{cc}
\frac{x}{c} & -\sqrt{1-\frac{x^{2}}{c^{2}}} \\
\sqrt{1-\frac{x^{2}}{c^{2}}} & \frac{x}{c}
\end{array}\right]
$$


Simplifying and combining then yields

$$
\mathbf{B}=\left[\begin{array}{c}
\frac{l_{w}}{2} \\
0
\end{array}\right]+c R(\delta)\left[\begin{array}{l}
0 \\
1
\end{array}\right]=\left[\begin{array}{c}
\frac{l_{w}}{2}-c \sqrt{1-\frac{x^{2}}{c^{2}}} \\
x
\end{array}\right]
$$

Removing the intermediate variables $c$ and $x$ then leaves

$$
\mathbf{B}=\left[\begin{array}{c}
\frac{l_{w}}{2}-\sqrt{\left[\left(2 r+l_{w}\right) \sin \left(\frac{d_{a}}{2 r+l_{w}}\right)\right]^{2}-\left[\left(r+\frac{l_{w}}{2}\right) \sin \left(\frac{2 d_{a}}{2 r+l_{w}}\right)\right]^{2}} \\
\left(r+\frac{l_{w}}{2}\right) \sin \left(\frac{2 d_{a}}{2 r+l_{w}}\right)
\end{array}\right]
$$

Assuming that the edge of the sensor beam is effective all the way to the right rear corner of the vehicle (point $\mathbf{D}$ in Figure 1), that point is derived as follows:

$$
\mathbf{D}=\mathbf{B}+R\left(\Delta-\frac{\pi}{2}\right) \frac{v_{w}}{2}\left[\begin{array}{l}
0 \\
1
\end{array}\right]
$$

where $v_{w}$ is the assumed width of the lead vehicle. The transformation matrix $R(\Delta-\pi / 2)$ can be simplified:

$$
R\left(\Delta-\frac{\pi}{2}\right)=\left[\begin{array}{cc}
\cos \left(\Delta-\frac{\pi}{2}\right) & -\sin \left(\Delta-\frac{\pi}{2}\right) \\
\sin \left(\Delta-\frac{\pi}{2}\right) & \cos \left(\Delta-\frac{\pi}{2}\right)
\end{array}\right]=\left[\begin{array}{cc}
\sin \Delta & \cos \Delta \\
-\cos \Delta & \sin \Delta
\end{array}\right]
$$

Hence the coordinate of the right rear corner of the lead vehicle in terms of basic parameters is given by

$$
\mathbf{D}=\left[\begin{array}{c}
\frac{l_{w}}{2}-\sqrt{\left[\left(2 r+l_{w}\right) \sin \left(\frac{d_{a}}{2 r+l_{w}}\right)\right]^{2}-\left[\left(r+\frac{l_{w}}{2}\right) \sin \left(\frac{2 d_{a}}{2 r+l_{w}}\right)\right]^{2}}+\frac{v_{w}}{2} \cos \left(\frac{2 d_{a}}{2 r+l_{w}}\right) \\
\left(r+\frac{l_{w}}{2}+\frac{v_{w}}{2}\right) \sin \left(\frac{2 d_{a}}{2 r+l_{w}}\right)
\end{array}\right]
$$

The other point of interest is then the point $\mathbf{E}$, which is the front middle of the following vehicle. This can be given as

$$
\mathbf{E}=\left[\begin{array}{c}
\frac{l_{w}}{2} \\
d_{a}-d
\end{array}\right]
$$

With the knowledge of the two points $\mathbf{D}$ and $\mathbf{E}$, we can determine the angle $\alpha$ between the center axis of the following vehicle and the right rear corner of the lead vehicle. More importantly, if we equate that angle to $\theta / 2$, we are determining the angle at which the lead vehicle departs the sensing region of the range sensor. Thus,

$$
\tan \frac{\theta}{2}=\frac{\left|\frac{l_{w}}{2}\right|+\left|\frac{l_{w}}{2}-\sqrt{\left[\left(2 r+l_{w}\right) \sin \left(\frac{d_{a}}{2 r+l_{w}}\right)\right]^{2}-\left[\left(r+\frac{l_{w}}{2}\right) \sin \left(\frac{2 d_{a}}{2 r+l_{w}}\right)\right]^{2}}+\frac{v_{w}}{2} \cos \left(\frac{2 d_{a}}{2 r+l_{w}}\right)\right|}{\left|d_{a}-d\right|+\left|\left(r+\frac{l_{w}}{2}+\frac{v_{w}}{2}\right) \sin \left(\frac{2 d_{a}}{2 r+l_{w}}\right)\right|}
$$


In this equation, the quantities $l_{w}$ and $v_{w}$, can be assumed to be constant values determined a priori. For particular circumstances, a value of $r$ can be chosen, although from the perspective of the ACC control logic, it must be expected that a range of curve radii can be encountered. The speed is not known ahead of time, and that has an impact on both the curve radius $r$ and the following distance $d$. Nevertheless, we posit that the most appropriate use of Eq. (15) is to choose values for all of these parameters, as well as for the sensor angle $\theta$, and then solve for $d_{a}$. Knowing $d_{a}$, one can then also determine $d_{l}=d-d_{a}$, which is the distance over which the car must travel after having lost the range signal, until it enters the curve and can then bend its sensor beam and re-acquire. Combined with the speed, this gives the time during which the vehicle is vulnerable to conditions outside the expectations of the sensing system. Equ. (15) cannot be solved for $d_{a}$ in closed form; in the examples that follow, we used the non-linear root finder in Matlab to solve the equation numerically.

\section{Numerical Example}

In this numerical example, we will solve for $d_{a}$ as a function of $v$, assuming that some of the other parameter values can be fixed, and the remaining variables can be chosen also to be single-valued functions of $v$. For example, we chose as standardized parameter values $l_{w}=12$ feet, and $v_{w}=7$ feet. In the latter case, this is the design vehicle width for a typical passenger car [7]. We chose $\theta=10$ degrees $=0.1745$ radians, which is the included angle for the center beam of the Omron AR211 unit used in Nissan ACC systems. Finally, we chose $r=800$ feet. This last choice was arbitrary but acceptable; the same analysis can be conducted with any other value.

The safe following distance maintained between vehicles when the ACC is in following mode can be represented by car-following stopping distance, which includes perception/reaction and braking distance and is given in consistent units by

$$
d=v t+\frac{v^{2}}{2 g(f+G)}
$$

where $t$ is the perception-reaction time for ACC system, which typically has a value of the order of $0.5 \mathrm{~s}$ [8]. This is a much shorter reaction time than is expected in manual driving, which is typically around 2-3 seconds [9]. The denominator of the $2^{\text {nd }}$ term of the right hand side of Eq. (16) contains all modifiers to the effect of gravity, including the coefficient of friction $f$ and the grade of the road $G$ in dimensionless form. If we assume $g$ is $32.2 \mathrm{ft} / \mathrm{sec}^{2}$, $\mathrm{f}$ is $0.30, \mathrm{G}$ is zero and $\mathrm{v}$ is $73.33 \mathrm{ft} / \mathrm{sec}$ (approximately $50 \mathrm{mph}$ ) for both vehicles in this example, then Eq. (16) yields $d=314.5$ feet. By moving all terms of Eq. (15) to one side of the equality, we turn the problem of solving for da into a root-finding exercise, which Matlab can do with standard numerical techniques. For the values given in this example, this yields $d_{a}=221.5$ feet. Thus, $d_{l}=d-d_{a}=93$ feet. At the speed of $73.33 \mathrm{ft} / \mathrm{sec}$, this means that the vehicle is "driving blind" for approximately 1.27 seconds. At least for the values given in this example, it seems wise to suggest that the ACC control unit pause at least a second or two before accelerating to the driver's desired speed, in order to distinguish between a situation where it is in fact safe to do so because the previously obstructing vehicle 
has left the lane in question, and a case where it only appears to be safe because of the effects of road curvature.

This advice, of course, comes from a worst-case safety perspective. It may be that certain aggressive drivers would become impatient with such a system. For example, the car might choose to wait until it can distinguish between a lane change and a curve transition, but the driver might know which was actually the case. Perhaps some "aggressiveness" factor could be tuned by the driver according to their preferences. As vehicle functions become increasingly automated, it is apparent that humanmachine interaction issues become more and more important, much the way user interface and ergonomics were with the last generation of automotive technology. Accordingly, this is an important area of research that should be pursued.

\section{Sensitivity Analysis}

We show how $d_{l}$ varies with marginal changes to other parameters such as the included angle of sensor, the radius of circular curve and vehicle speed, using as our nominal state the data from the analysis. Because of the number of dimensions involved, we cannot hope to completely characterize the behavior of Eq. (15) with this analysis, but it can serve to illustrate the shape of its partial derivatives, and reinforce the reasonableness of these relationships.

The relation between $d_{l}$ and $\theta$ is approximately linear in this range of values, as shown in Fig. 2. Sensors with included angles between 4 and 12 degrees are considered. As the angle of sensor increases, the $d_{l}$ decreases which means that the blind time is decreasing. However, we have to make sure that increasing the sensor angle increases the likelihood of false readings from adjacent lanes, particularly at curves.

While we chose a specific value of the curve radius $r$ for the example, it is also fair to say that it depends in some measure on the speed $v$, which is presumably within the range of the design speed for the facility in question. It is not reasonable, however, to tie Eq. (15) to a specific relationship between speed and curve radius, since the vehicles are not necessarily traveling at the design speed, and because any such relation only gives a minimum value of the curve radius anyway. Beyond that, ample room is left for trading off curve radius versus superelevation, and designers can always choose larger-than-necessary curve radii to improve comfort, aesthetics, and other considerations. Fig. 3 shows how a range of curve radii affects the computation of $d_{l}$.

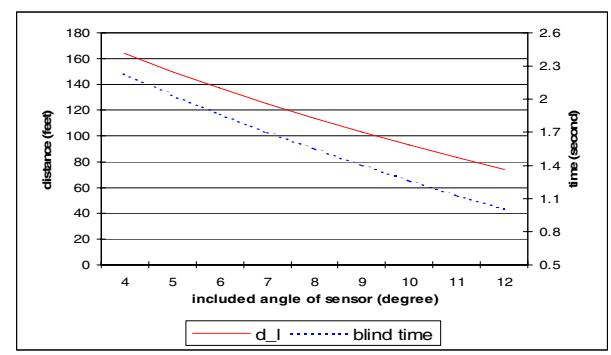

Fig. 2. Effect of the range sensor included angle

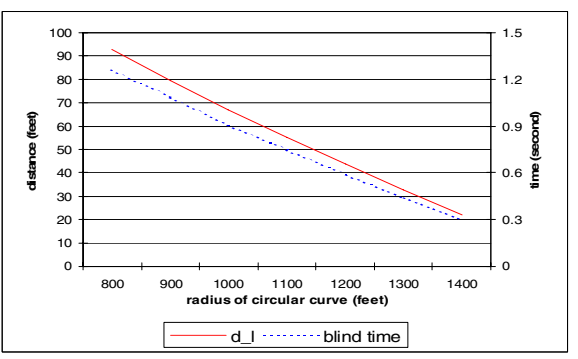

Fig. 3. Effect of curve radius 
All radii are greater than the minimum required for a speed of $50 \mathrm{mph}$. With the same speed, but decreasing curvature, the lead vehicle does not deviate from the following vehicle's axis as drastically, so the blind time decreases.

Fig. 4 shows how $d$ and $d_{l}$ are affected by $v$, via Eq. (15) and (16), together with factors to transform speed to the more customary units of miles per hour. We chose the minimum curve radius for each speed range and safe following distance as increasing functions of velocity. Intuitively, blind time should decrease with curve radius, but increase with stopping distance. It is clear from this figure that the latter effect is more pronounced, so the blind time increases. Of course, there are limits to the range of the range sensor.

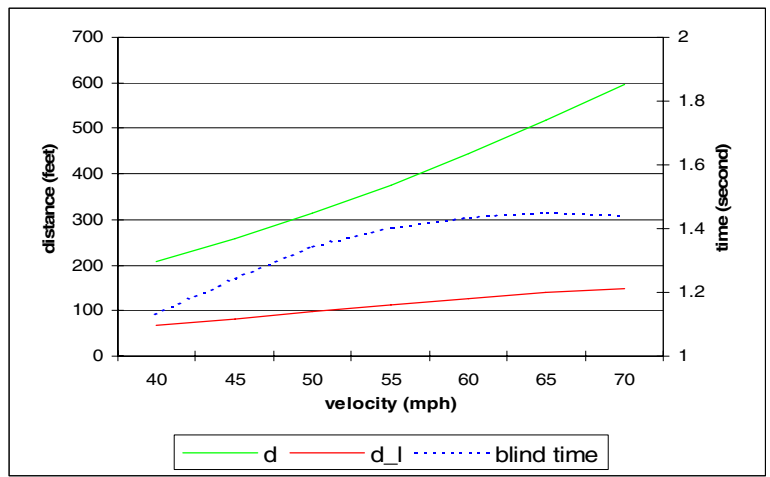

Fig. 4. Effect of the velocity of the lead vehicle

\section{Conclusion}

In this paper we have described and illustrated a situation where an autonomous cruise control in car-following mode may inadvertently lose track of the lead vehicle, and may mistake that loss of target as being clear to accelerate. This is a potentially dangerous conclusion, since the lead vehicle has not changed lanes in this scenario, and will re-appear within the sensor's "vision" as soon as the following vehicle knows it is on a curve and rotates the sensor beam appropriately. We were not the first to discuss or even mitigate against this problem, but our presentation of the geometric model of this situation is original to our knowledge.

Using realistic example numbers, it is shown that the time lag between losing and re-acquiring the lead vehicle target is relatively small on the human scale, and therefore it might be wise not to employ ACC scenarios that over-react within time windows this small. There is a trade-off, however, because the ACC would be forced to behave this way in safe situations as well. This "sluggishness" might downgrade the user's perceived convenience of ACC, which is the ability to quickly get underway again at the desired speed once potential threats have moved aside.

It is not necessarily true that this issue will remain forever; however, it is probably not within the limits of range-sensing technology alone to solve the problem. The addition and fusion of a second sensor input, such as precise geolocation and an accu- 
rate underlying map database, or an intelligent vision system that can recognize the presence and curvature of circular arcs ahead, might allow the following vehicle to react to curvature that the lead vehicle is experiencing even before it is in the curve itself. This is the approach being followed in the ongoing Automotive Collision Avoidance System (ACAS) field operational test conducted by General Motors, and presumably by a number of other manufacturers as well.

\section{References}

1. Domsch, C. and D. Sandkuhler (2000). Test procedures for ACC-sensors. Proceedings of the $7^{\text {th }}$ World Congress on Intelligent Transport Systems, pp. 1-8.

2. Jagtman, H.M. and E. Wiersma (2003). Driving with adaptive cruise control in the real world. $16^{\text {th }}$ ICTCT Workshop, pp. 1-8.

3. General Motors Corporation (2002). Phase I Interim Report: Automotive Collision Avoidance System Field Operational Test. Report No. DOT HS 809 454, National Highway Traffic Safety Administration, Washington, D.C.

4. Fancher, P., R. Ervin, J. Sayer, M. Mefford, and J. Haugen (1998). Intelligent Cruise Control Field Operational Test (final report). University of Michigan Transportation Research Institute (UMTRI), Ann Arbor, MI.

5. Lovell, D. J. (1999). Automated Calculation of Sight Distance from Horizontal Geometry. ASCE Journal of Transportation Engineering Vol. 125, No. 4, pp. 297-304.

6. Lovell, D. J., J.-C. Jong, and P. C. Chang (2001). Improvements to Sight Distance Algorithm. ASCE Journal of Transportation Engineering Vol. 127, No. 4, pp. 283-288.

7. AASHTO (2001). A Policy on Geometric Design of Highways and Streets. American Association of State Highway and Transportation Officials, Washington, D.C.

8. Rajamani, R. and C. Zhu (2002). Semi-autonomous Adaptive Cruise Control Systems. IEEE Tran. on Vehicular Technology, Vol. 51, No. 5, pp. 1186-1192.

9. Roess, R. P., W. R. McShane, and E. S. Prassas (1998). Traffic Engineering, $2^{\text {nd }}$ edition. Pearson, New York, NY. 